\title{
Probing the shape of atoms in real space
}

\author{
M. Herz, F. J. Giessibl,* and J. Mannhart ${ }^{\dagger}$ \\ Universität Augsburg, Institute of Physics, Electronic Correlations and Magnetism, Experimentalphysik VI, \\ Universitätsstrasse 1, D-86135 Augsburg, Germany
}

(Received 7 March 2003; published 1 July 2003)

\begin{abstract}
The structure of single atoms in real space is investigated by scanning tunneling microscopy. Very high resolution can be obtained by a dramatic reduction of the tip-sample distance. The instabilities which are normally encountered while using small tip-sample distances are avoided by oscillating the tip of the scanning tunneling microscope vertically with respect to the sample. The surface atoms of $\mathrm{Si}(111)-(7 \times 7)$ with their well-known electronic configuration are used to image individual samarium, cobalt, iron, and silicon atoms. The resulting images resemble the charge density corresponding to $4 f, 3 d$, and $3 p$ atomic orbitals.
\end{abstract}

DOI: 10.1103/PhysRevB.68.045301

PACS number(s): 68.37.Ef, 68.47.Fg, 68.37.Ps

Democritos of Abdera (460 B.C. -370 B.C.) predicted the existence of atoms and speculated that atoms come in different sizes and shapes. ${ }^{1}$ Today, we know that the size and shape of an atom depends on its chemical species and the way it is bonded to neighboring atoms. Atoms consist of the small, positively charged nucleus and electrons with negative charge $-e$ which orbit the nucleus with a speed of a few percent of the speed of light. Because of the uncertainty principle of quantum mechanics, articulated by Werner Heisenberg in 1927, we cannot observe single electrons within their orbit. However, quantum mechanics specifies the probability of finding an electron at position $\mathbf{x}$ relative to the nucleus. This probability is determined by $|\psi(\mathbf{x})|^{2}$, where $\psi(\mathbf{x})$ is the wave function of the electron given by Schrödinger's equation. ${ }^{2}$ The product of $-e$ and $|\psi(\mathbf{x})|^{2}$ is usually interpreted as charge density, because the electrons in an atom move so fast that the forces they exert on other charges are essentially equal to the forces caused by a static charge distribution $-e|\psi(\mathbf{x})|^{2}$. For the electrons of an atom, the solutions to Schrödinger's equation are wave functions $\psi_{n \operatorname{lm} \sigma}(\mathbf{x})$ defined by four quantum numbers: the principal quantum number $n$, the angular momentum $l$, the $z$ component of angular momentum $m$, and the spin $\sigma .^{3}$

The shape of atoms, as given by their total charge density, can be determined by $\mathrm{x}$-ray or electron scattering if they are arranged periodically in a crystal. X-ray scattering allows one to measure the roughly spherical charge density of ions in alkali-metal halides ${ }^{4}$ or the nonspherical structure of atoms crystallized in a diamond lattice. ${ }^{5}$ Recently, $d$-orbital holes were found in the charge density of copper-oxide compounds by transmission electron microscopy measurements. ${ }^{6}$ Observing the charge density of single atoms in real space became feasible in 1982 with the invention of scanning tunneling microscopy (STM). ${ }^{7}$ In contrast to the techniques listed above, STM does not probe the total charge density of an atom, but the charge density at the Fermi level $\rho_{\text {Fermi }} .{ }^{8,9}$ For a negative sample bias voltage, the charge density of the energetically highest occupied electron states is imaged, while for a positive sample bias, the charge density of the lowest unoccupied states is imaged. If the states at the Fermi level have an $s$-type symmetry, the constant-density surface $\rho_{\text {Fermi }}(\mathbf{x})=c$ is composed of spheres centered at the nuclei of the surface atoms. If $\rho_{\text {Fermi }}$ is obtained from atomic states with higher angular momentum, $\rho_{\text {Fermi }}$ can have a more complex shape, in particular, for large values of $\rho_{\text {Fermi }}$ in close proximity to the atoms. Thus, if higher-momentum states are present at the Fermi energy, the corresponding STM images are expected to reflect their shape, such that the shapes of atomic images are nonspherical and display orbital substructures. However, it is important to note that orbitals in the sense of mathematical constructs with an amplitude and phase cannot be observed, but the density of charges within a certain energy range corresponding to specific orbitals, $\mathrm{see}^{8,9}$ and the discussion ${ }^{10}$ and references therein.

In spite of the large amount of standard STM data gathered, the observation of atomic substructures linked to atomic higher-momentum states has not been reported, instead STM images of atoms always appear more or less parabolic. Interestingly, tunneling channels originating from higher orbital momentum states have been found in tunneling experiments involving mechanically controllable breakjunction techniques, ${ }^{11}$ where tunneling gap widths of the order of bulk next-neighbor distances can be realized. However, imaging is not possible with the break-junction technique. While "subatomic" resolution, showing two maxima in the image of a single atom linked to $\mathrm{sp}^{3}$-type orbitals, has been observed by force microscopy ${ }^{12,13}$ features within single atoms have not yet been observed by STM. After a presentation of the work reported in Ref. 12 by a member of our team, Doyen from the University of Munich insisted that orbital structures should be visible in STM, if these structures can be imaged by force microscopy. Also, Chen (Chap. 7 in Ref. 9) discusses the similarity of the functional dependence of the tunneling current and the attractive part of the tip-sample force. These theoretical arguments and the experimental appearance of higher-momentum states in atomic force microscopy (AFM) and break-junctiontunneling experiments inspired us to think about possible reasons for the absence of subatomic resolution in the standard STM. ${ }^{13}$

One likely cause is the large tip-sample separation in the standard STM $(0.5-0.8 \mathrm{~nm})$. Even if the Fermi level in a sample or tip was occupied with higher-momentum states, the contour lines of the electron density show at this large distance only one single maximum per surface and tip atom. A second possible cause is the geometry of the electronic 
states participating in the tunneling process. The valence bands of solids can often be constructed from atomic states by linear combination of atomic orbitals. As a consequence, spatial features are expected to be present in individual tunneling channels. ${ }^{9}$ Because of various effects, ${ }^{3}$ the energy of atomic (and bulk) states depends on the angular momentum $l$. Thus, if large tunneling voltages are used, many states can contribute to the tunneling current, blurring out spatial features. We concluded that small bias voltages and very small tip-sample distances are required for enhanced resolution.

In the standard STM, the tip-sample distance decreases when the tunneling impedance, given by the bias voltage divided by the set point of the tunneling current is decreased. In STM operation, choosing the set point of the tunneling impedance implies a trade-off between noise-level and tipload. If the set point of the tunneling current is too small, the corrugation in the image is small and the noise level is high. If the current set point is too high (and/or bias voltage too low), the tip-sample forces become excessive and destruction of tip and sample occurs while scanning at high loading forces. However, when the lateral scan is stopped to allow for current-versus-distance spectroscopy experiments, small tip-sample distances can be realized without tip or surface destruction. A recent theoretical study ${ }^{15}$ has shown that single-atom tips can withstand repulsive forces up to $30 \mathrm{nN}$ without damage. However, pushing the tip laterally over a surface at high loading forces damages both the tip and the sample. A stop-and-go approach, where the scan is stopped, the tip is lowered until it reaches its high current set point, retracted, and moved laterally to start all over again would remedy this situation. We found experimentally that a much simpler and faster approach is possible by deliberately vibrating the tip vertically (see Fig. 1). Because of the strong distance dependence of the tunneling current, the current only flows when the tip is close to its lower turnaround point. The lateral motion of the tip during this time when it is very close to the sample is given approximately by the scanning speed times that are less than half the oscillation period, amounting to only a few picometers.

A similar concept already proved successful in tapping force microscopy, where the oscillation of the tip also reduces lateral forces and decreases tip wear. ${ }^{14}$ The tip oscillation in dynamic STM reduces or even prevents the wear which normally occurs when scanning a probe tip across a surface with repulsive load forces.

In our experiments, the vibration of the tip is done by mounting it on a quartz force sensor which oscillates at frequency $f_{0} \approx 20 \mathrm{kHz}$, as described in detail in Ref. 16. While the oscillation could also be performed with a standard STM, mounting the tip on a cantilever allows one to monitor two more important observables: the conservative and the dissipative components of the tip-sample force. The forces which occur during tunneling have been measured before with a different technique, albeit not with atomic resolution. ${ }^{17}$ Measuring the conservative force component yields information about the tip-sample distance. Monitoring the dissipative component allows one to assess if tip and sample atoms are deflected strongly from their equilibrium positions. ${ }^{19}$ The STM images are recorded in the topographic mode, where

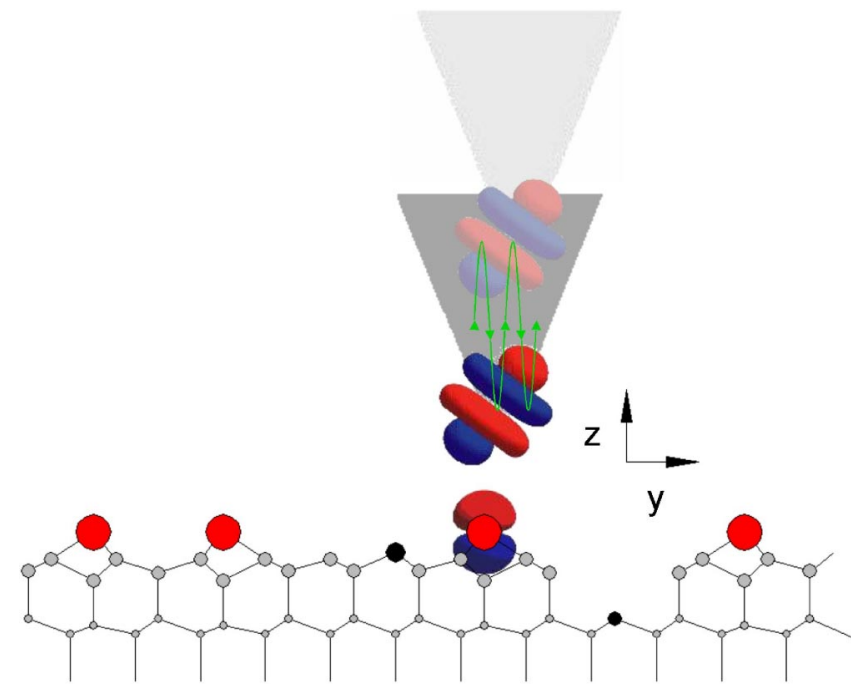

FIG. 1. (Color online) Principle of the dynamic STM. The tip is mounted on a quartz cantilever (not shown here, see (Ref. 16) for details) with a stiffness of $1800 \mathrm{~N} / \mathrm{m}$ which oscillates at a fixed amplitude $A \approx 0.3 \mathrm{~nm}$. The unperturbed resonance frequency is $f_{0}$ $\approx 20 \mathrm{kHz}$. Conservative components in the tip-sample force are measured by a change in the cantilever's eigenfrequency that they are causing (Ref. 18). Nonconservative components in the tipsample force are measured by monitoring the dissipation energy which has to be supplied to the cantilever to maintain a constant amplitude (Ref. 19).

the average tunneling current (averaged over a time of approximately 30 oscillation cycles) is kept constant by adjusting the $z$ position of the tip accordingly. For an exponentially decaying tunneling current $I(z)=I_{0} \exp (-2 \kappa z)$, the average tunneling current $I_{a v}$ is smaller than the peak tunneling current $I_{p k}$ with $I_{a v} / I_{p k} \approx(2 \pi \kappa A)^{-0.5} \cdot{ }^{18}$ In this paper, we present measurements performed by dynamic STM and report the observation of atomic images containing symmetry features linked to $p, d$, and $f$ orbitals of $\mathrm{Si}, \mathrm{Co}$, and $\mathrm{Sm}$ atoms. All experiments have been performed in ultrahigh vacuum at a pressure of $p \approx 10^{-8} \mathrm{~Pa}$ and ambient temperature $T \approx 300 \mathrm{~K}$.

In STM, the role of tip and sample is perfectly interchangeable-the image is a convolution of tip and sample states. For zero temperature, the tunneling conductivity depends on the matrix elements $M_{\mu \nu}$ in a first-order calculation as

$$
\frac{I_{t}}{V}=(2 \pi)^{2} G_{0} \sum_{\mu \nu} D_{S \mu}\left(E_{F}\right) D_{T \nu}\left(E_{F}\right)\left|M_{\mu \nu}\right|^{2}
$$

with the conductance quantum $G_{0}=2 e^{2} / h$. Here, the densities of states $D_{S \mu}$ and $D_{T \nu}$ of the corresponding band states of sample and tip as well as the matrix elements $M_{\mu \nu}$ are assumed to be constant in the energy range $\mathrm{eV}$ around the Fermi level $E_{F}$.

In Bardeen's treatment of a tunneling junction, ${ }^{9}$ the matrix elements $M_{\mu \nu}$ are evaluated to be 

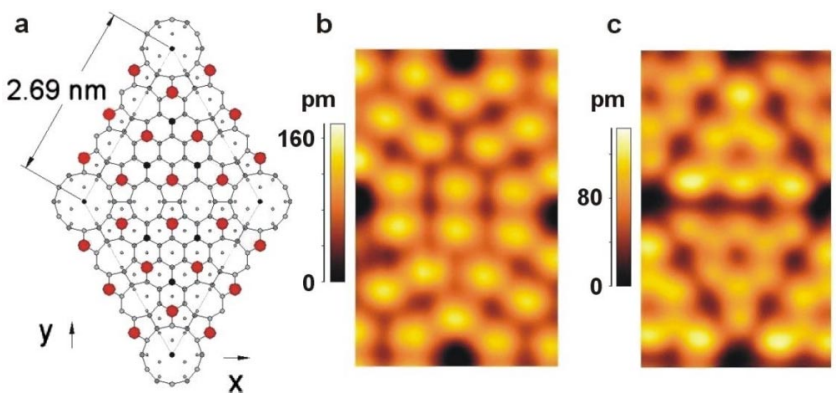

FIG. 2. (Color online) (a) Surface unit cell of $\mathrm{Si}(111)-(7 \times 7)$ after the dimer-adatom-stacking fault model. The diamond-shaped unit cell has characteristic features: deep corner holes at the corners of the diamond (see Fig. 1 for a cross section of the corner hole along the long diagonal of the unit cell), 18 dimer atoms, 12 adatoms, and 6 restatoms. One triangular halve of the unit cell develops a stacking fault. The surface atoms (adatoms) are printed in red. Each adatom is bonded to the layer underneath by three covalent bonds. The fourth valence electron forms a dangling bond $\left(3 s p^{3}\right.$ state), which sticks out perpendicular to the surface. The rest atoms, printed in full black circles, are located more than $100 \mathrm{pm}$ below the adatoms. (b) The standard STM image of the empty states on the $\mathrm{Si}(111)-(7 \times 7)$ surface (sample bias $2.4 \mathrm{~V}$, tunneling current 1 nA). (c) The standard STM image of the filled states on the Si(111)- $(7 \times 7)$ surface (sample bias $-2.4 \mathrm{~V}$, tunneling current 1 $\mathrm{nA})$. In the filled state image, the upper half of the diamond-shaped unit cell appears to be slightly higher than the lower half, caused by the stacking fault. In images $b$ and $c$, the image of a single adatom is a paraboloid with a radius of $\approx 0.5 \mathrm{~nm}$. Ir tips have been used for both images.

$$
M_{\mu \nu}=-\frac{\hbar^{2}}{2 m_{e}} \int_{\Omega_{T}}\left(\chi_{\nu}^{*} \nabla^{2} \psi_{\mu}-\psi_{\mu} \nabla^{2} \chi_{\nu}^{*}\right) d V
$$

with the electron's mass $m_{e}$, and tip and sample states $\chi_{\nu}$ and $\psi_{\mu}$, respectively. The integration extends over the tip volume $\Omega_{T}$, see, e.g., Chap. 2 in Ref. 9. The tunneling process is considered to be spin independent for the case of a nonmagnetic tip and/or sample. The modulus of the matrix element does not change if tip and sample states switch roles, giving rise to the reciprocity principle: If the electronic states of the tip and the sample under observation are interchanged, the image should be the same-see p. 88 in Ref. 9. Thus, a STM image can either be interpreted as one image of $N$-sample atoms probed by the front atom of the tip or as $N$ images of a front atom probed by $N$-sample atoms. In this study, we are interested in the image of the front atom of the tip. Therefore, the electronic state of the sample atoms needs to be known. We have chosen a $\operatorname{Si}(111)-(7 \times 7)$ surface as the sample [see Fig. 2(a)], because the electronic states of the surface atoms (adatoms) in $\mathrm{Si}(111)-(7 \times 7)$ are precisely characterized, so that the tip states can be inferred from the images.

Each adatom of the $\mathrm{Si}(111)-(7 \times 7)$ surface exposes one $s p^{3}$-dangling bond oriented perpendicular to the surface. The large distance between adjacent adatoms (at least $0.67 \mathrm{~nm}$ ) allows one to clearly separate individual tip atom images. The shape and the apex radius of the images are used to characterize the electronic states of the tip. The advantage of
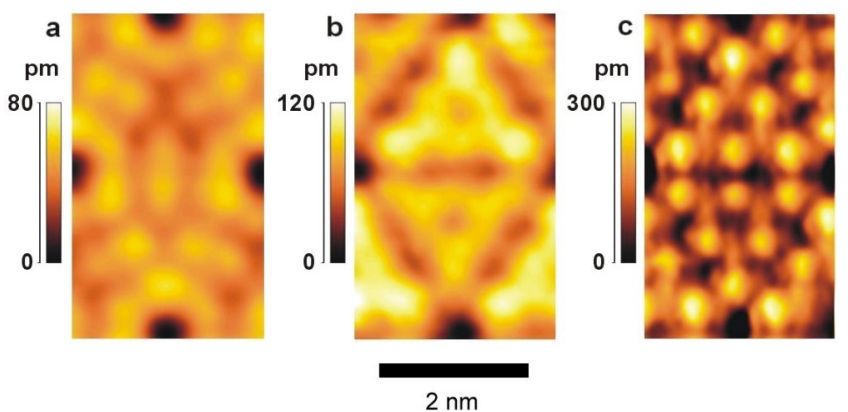

FIG. 3. (Color online) (a) STM images of a $\mathrm{Si}(111)-(7 \times 7)$ surface taken with a Sm tip. A standard STM with sample bias $2 \mathrm{~V}$, $I_{t}=100 \mathrm{pA}$; (b) sample bias $-2 \mathrm{~V}, I_{t}=200 \mathrm{pA}$; and (c) a dynamic STM with sample bias $-1 \mathrm{~V}, I_{a v}=100 \mathrm{pA}, A=0.8 \mathrm{~nm}$.

scanning an area containing many sample atoms is that the potential errors due to multitip effects are easily detected. The observation of single defects on the Si surface rules out double-tip or multitip effects, and the observation of deep corner holes offers a proof that the tip is pointed and sharp. This is not a restriction of our method, because, in principle, well-defined tips can also be created ${ }^{13}$ and unknown sample states could be studied with these tips. As a reference, standard STM images of Si(111)-(7×7) are shown in Fig. 2. Figure 2(a) shows the structure of the $\mathrm{Si}(111)-(7 \times 7)$ surface and Figs. 2(b) and 2(c) are STM images taken with an iridium tip, using a tunneling current of $1 \mathrm{nA}$ and a sample bias of $+2.4 \mathrm{~V}$ (empty states) in Fig. 2(b), and a sample bias of $-2.4 \mathrm{~V}$ (filled states) in Fig. 2(c). The apex of the adatom image can always be approximated as a paraboloid. The topographic data around the peak is approximated with $z(x, y)=z_{0}-\left(x-x_{\text {peak }}\right)^{2} / 2 R_{x}-\left(y-y_{\text {peak }}\right)^{2} / 2 R_{y}$, thus $R_{x}$ and $R_{y}$ are easily determined from the topographic data. Application of this formula to the measured profiles in Figs. 2(b,c) yields apex radii of $0.5 \mathrm{~nm}$.

Figure 3 shows the difference between imaging in the standard STM and the dynamic STM. A samarium tip was used to image Si in the standard STM in Figs. 3(a) and 3(b), and the dynamic STM in Fig. 3(c). Because Sm is a relatively soft material, the tip can only withstand a small tunneling current of $100 \mathrm{pA}$ at a sample bias of $2 \mathrm{~V}$ in the standard STM. In the dynamic STM with $A=0.8 \mathrm{~nm}$, the average current can be set to $100 \mathrm{pA}$ (yielding a peak current $\left.I_{P k} \approx 700 \mathrm{pA}\right)$ at a bias of $-1 \mathrm{~V}$. Thus, the dynamic STM allows one to get much closer to the surface without tip degradation, resulting in improved spatial resolution. The curvature of a STM image of a single atom is a characteristic feature. According to a calculation by Chen, ${ }^{9}$ the radius of the atom images is a function of the distance $z$ between the tip and sample atoms and the type of atomic tip and sample states:

$$
\begin{gathered}
s-s: R=z, \\
s-p_{z}: R=\frac{z}{1+1 / \kappa z}, \\
p_{z}-d_{z}: R=\frac{z}{1+4 / \kappa z}
\end{gathered}
$$



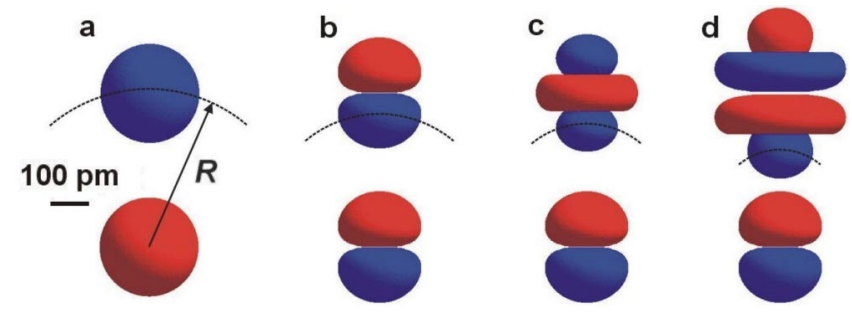

FIG. 4. (Color online) (a) Apparent radius $R$ of a surface atom in the standard STM mode as a function of tip and sample states for a tip-sample distance of $z=0.4 \mathrm{~nm}$. Calculated with Eqs. (3)-(6) after Sec. 6.2 in Ref. 9. The tunneling current is assumed to decay with distance $d$ as $\exp (-2 \kappa d)$, with $\kappa=10 \mathrm{~nm}^{-1}$. (a) Tip state, $s$ orbital, sample state, $s$ orbital. The apparent radius of the adatom image is given by the distance, thus $R_{a}=0.4 \mathrm{~nm}$. (b) Tip state, $p_{z}$ orbital; sample state, $p_{z}$ orbital; $R_{b}=0.27 \mathrm{~nm}$. (c) Tip state, $d_{z^{2}}$ orbital; sample state, $p_{z}$ orbital, $R_{c}=0.2 \mathrm{~nm}$. (d) Tip state, $f_{z^{3}}$ orbital; sample state, $p_{z}$ orbital; $R_{d}=0.15 \mathrm{~nm}$.

$$
p_{z}-f_{z}: R=\frac{z}{1+7 / \kappa z},
$$

where $\kappa$ is the decay constant of the tunneling current with a typical value of $\kappa=1 \AA^{-1}$.

Figure 4 shows four combinations of the tip and sample atomic states. If the tip and sample states are $s$ type, the radius of the atom image is simply the distance between the tip- and the sample atom. The higher the angular momentum is, the smaller is the radius of the atom images. Sharply peaked atom images are observed with tip states with a large angular momentum. It is evident from Fig. 4 that the angular orientation of the tip and sample orbitals is important. Figure 4 displays only tip orbitals oriented in the $z$ direction, while the two other $p$-orbital orientations $p_{x}, p_{y}$, the four other $d$-orbital orientations $d_{x y}, d_{x z}, d_{y z}, d_{x^{2}-y^{2}}$, and the six other $f$-orbital orientations can also contribute to the tunneling current. However, it can be shown that the matrix element between a $p_{z}$ sample and $p_{z}, d_{z^{2}}$, and $f_{z 3}$ tips is much greater than the matrix element between a $p_{z}$ sample and the orbitals that are not aligned in the $z$-direction.

An experimental test of Eqs. (3)-(6) can be performed while imaging a surface with known electronic states with a tip that is also characterized by a known state. On the $\mathrm{Si}(111)-(7 \times 7)$ surface, the sample states are well approximated by the $p_{z}$ states. The tip states depend on the chemical identity of the tip atom. We have, therefore, examined for tip materials which exhibit $p, d$, and $f$ states. Si with an electronic configuration of $[\mathrm{Ne}] 3 s^{2} 2 p^{2}$ is a good choice for $p$ states, because the $3 p$ states are the highest occupied orbitals in $\mathrm{Si}$. Co with an electronic configuration of $[\mathrm{Ar}] 3 d^{7} 4 s^{2}$ is expected to have a $d$ symmetry and rare-earth elements are excellent candidates for $f$-type tip atoms. We have chosen $\mathrm{Sm}$ (electronic configuration [Xe] $4 f^{6} 6 s^{2}$ ) for an $f$-type tip, because it is one of the most stable rare-earth elements. For imaging with very small tip-sample distances, large forces are expected to occur and mechanical stiffness of the STM tips is important. Rare-earth elements are interesting for STM studies, because in contrast to hydrogen and other lighter atoms, the $6 s$ electrons are bonded more strongly to
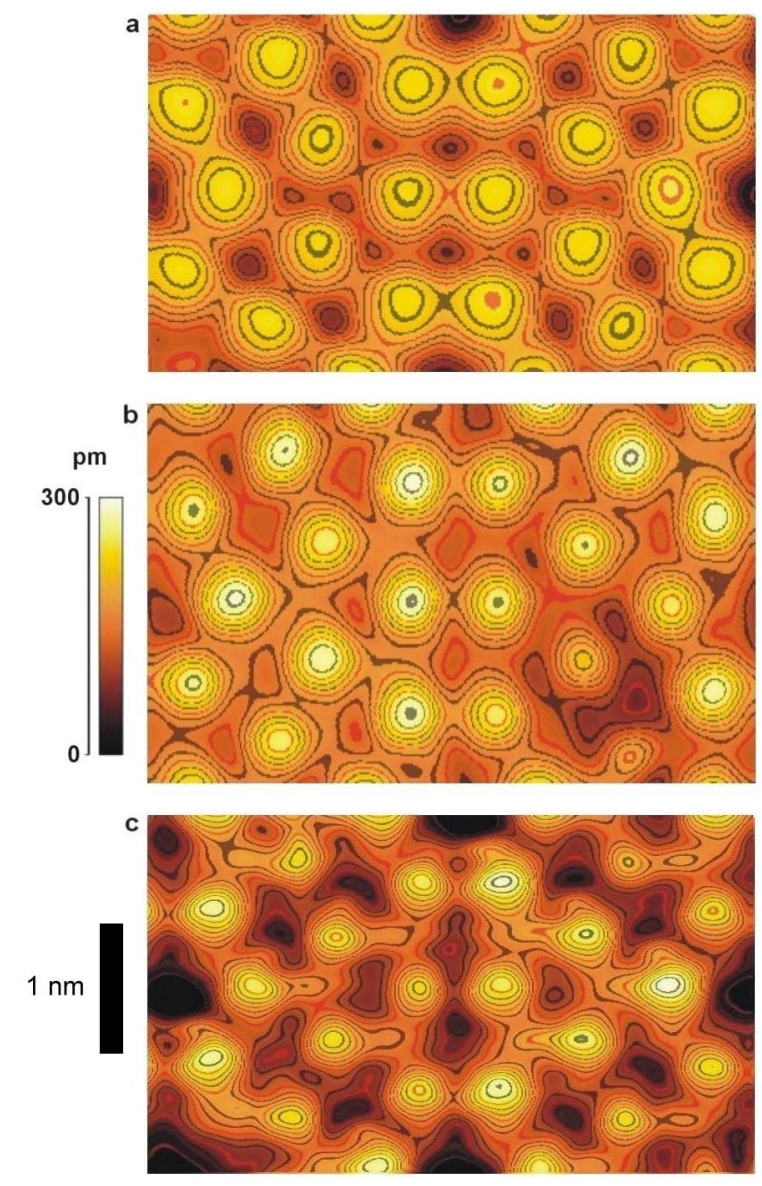

FIG. 5. (Color online) Dynamic STM images of the $\mathrm{Si}(111)-(7 \times 7)$ surface showing the influence of the angular momentum number $l$ of the tip state on the apex radii. The images have been observed with one instrument, using similar imaging parameters but tips made of different materials. The spacing of the contour lines is $20 \mathrm{pm}$ and their increasing density from (a)-(c) is caused by decreasing apex radii. (a) Image taken with a Si tip (a Fe tip suffered a mild collision with a Si sample, leading to a Fe tip covered with a Si cluster). Sample bias $300 \mathrm{mV}$, tunneling current $I_{a v}=300 \mathrm{pA}$ with $A=0.1 \mathrm{~nm}$. The tip atom is presumably in a $p_{z}$-like $s p^{3}$ state $(l=1)$. The experimental apex radius is $\approx 0.5 \mathrm{~nm}$. (b) Image taken with a Co tip at a sample bias of $200 \mathrm{mV}$, tunneling current $I_{a v}=100 \mathrm{pA}$ with $A=0.5 \mathrm{~nm}$. The image is expected to originate from a convolution of a Co $3 d_{z^{2}}$ state $(l=2)$ with the $p_{z}$ $\mathrm{Si}$ states. The apex radius is $\approx 0.2 \mathrm{~nm}$. Rest atoms are visible in the left half of the unit cell. (c) Image taken with a Sm tip [see also Fig. 3 (c)]. The rest atoms are clearly separated from the adatoms. The apex radius is only $0.14 \mathrm{~nm}$. The tilt angle of the $f_{z^{3}}$ state is presumably close to $0^{\circ}$. Other states $(4 f, 6 s)$ are possibly contributing to the image, too.

the nucleus than the $4 f$ electrons. Thus, the $4 f$ electrons participate in electronic conduction and in the tunneling process from the tip to the sample. However, the average distance to the nucleus is larger for the $6 s$ electrons than for the $4 f$ electrons. Imaging Sm by STM implies that the outer $6 s$ electron shell has to be penetrated-literally requiring to look into the inner parts of an atom.

Figure 5 displays the series of dynamic STM images of $p$, $d$, and $f$ atoms, realizing the three cases presented in Figs. 
4(b)-4(d). In Fig. 5(a), a Si front atom is imaged by the $\mathrm{Si}$ surface, in Fig. 5(b), a Co atom; and in Fig. 5(c), a Sm atom. As revealed by this image, with increasing angular momentum of the tip states, the apex radii decrease indeed, in a similar way as predicted in Eqs. (4)-(6). Figure 5(c) is recorded with a tip made of pure Sm. The clear visibility of the rest atoms and the enhanced corrugation together with the very small apex radius of $\approx 0.14 \mathrm{~nm}$ prove the angular confinement resulting from $4 f_{z^{3}}(l=3)$ tip states. Rest atoms have already been observed by force microscopy. ${ }^{13,20,21}$

Figure 6(a) shows an experiment where a CoSm rareearth magnet mounted on a quartz force sensor was used as a tunneling tip. The composition of the tip was $\mathrm{Co}_{6} \mathrm{Fe}_{3} \mathrm{Sm}$, as determined by the energy dispersive $\mathrm{x}$-ray analysis. The magnetic-field axis was oriented perpendicular to the sample surface. The eigenfrequency of the force sensor was $f_{0}$ $=19621 \mathrm{~Hz}$, and the positive frequency shift which was measured during imaging implies repulsive tip-sample forces during imaging. Qualitatively, the adatom images in Fig. 6(a) can be explained with a $p_{z}$-sample which tunnels into an $f$ tip state tilted by an angle $\Theta$ as shown in Fig. 6(b). Repulsive forces are expected to occur when the 6s shell in Sm has to be penetrated in order to allow for a large tunneling current. A comparison of this data with images taken with pure $\mathrm{Sm}, \mathrm{Co}$, and $\mathrm{Fe}$ tips suggests that the tip atom in this image was $\mathrm{Sm}$. The crescent surrounding the cusp is expected to originate from the upper hoop surrounding the club of an $f_{z^{3}}$ orbital as shown in Fig. 6(b). Several investigations were performed to check if these images are caused by experimental artifacts. Interference due to a multitip image has to be ruled out because of the presence of a single atomic surface defect indicated by a green arrow in Fig. 6(a) and because of the large depth of the corner hole image. Further, rotating the fast scanning direction and varying the scanning speed did not change the images, which rules out feedback artifacts. The cantilevers frequency shift and damping were recorded in parallel to the topography record. The frequency shift according to Fig. 6(a) was positive, i.e., repulsive forces have been acting. The unusually sharp apex radii cannot be explained by atomic relaxations due to tip-sample forces. While the radius of the atomic images decreases in STM when attractive tip-sample forces act, ${ }^{22}$ imaging at repulsive forces leads to increasing radii of the atomic images, as shown experimentally in Figs. 15 and 16 in Ref. 13. In the experiments presented here, repulsive tip-sample forces were acting which cause even an increase in the experimental apex radii. The damping signal did not show significant variations within the adatom image, which proves that a hysteretic lateral jump of the adatom or the tip atom, which possibly could have explained the sharp atomic peaks, did not occur. ${ }^{19}$ In theory, fluctuations of the cantilevers amplitude could explain modulations in topography. However, the oscillation amplitude was kept constant by an amplitude control circuit and monitored, ruling out amplitude fluctuations as a source of the measured atom shapes.

There is also quantitative theoretical evidence that the observed features are images of orbitals. Figure 6(c) is a magnified view of an experimental single adatom image acquired with the CoSm tip in the dynamic STM. Figure 6(d) shows a
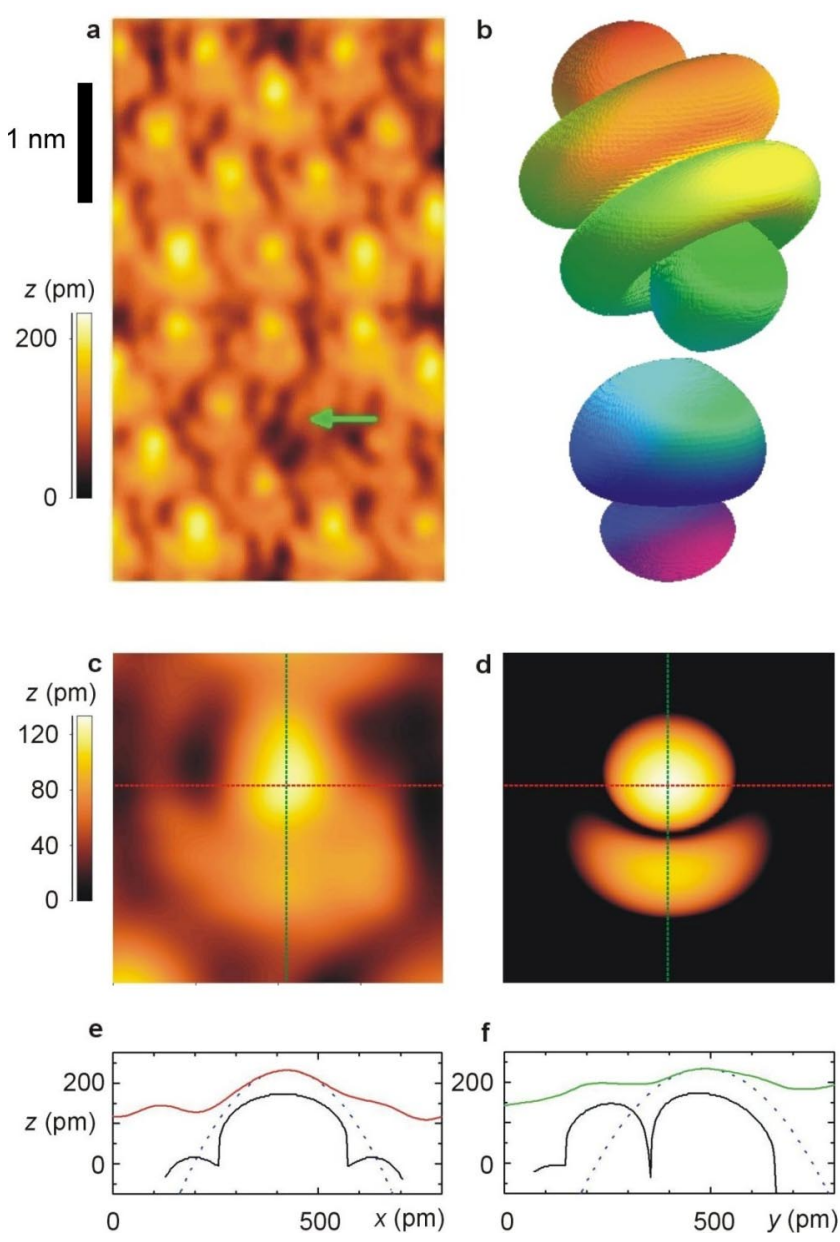

FIG. 6. (Color online) (a) Ultrahigh resolution image of a $\mathrm{Si}(111)-(7 \times 7)$ surface, acquired by dynamic STM with a $\mathrm{Co}_{6} \mathrm{Fe}_{3} \mathrm{Sm}$ tip oscillating with an amplitude of $A=0.5 \mathrm{~nm}$ at a sample bias voltage of $-100 \mathrm{mV}$ and an average tunneling current $I_{a v}=200 \mathrm{pA}$. The green arrow indicates an atomic defect (missing center adatom). (b) Schematic presentation of the current carrying atomic states leading to the observed image shown in (a). The $s p^{3}$ silicon states are tunneling mainly into a $\mathrm{Sm} 4 f_{z^{3}}$ tip state tilted by a fixed angle, determined to be $\approx 37^{\circ}$. (c) Experimental image of a single $\mathrm{Si}$ adatom imaged with a $\mathrm{Co}_{6} \mathrm{Fe}_{3} \mathrm{Sm}$ tip. Average tunneling current $I_{a v}=1 \mathrm{nA}$, sample bias: $-100 \mathrm{mV}$, amplitude $A$ $=0.5 \mathrm{~nm}$. (d) Calculated dynamic STM topography image for a silicon $3 p_{z}$ sample state and a $\mathrm{Sm} 4 f_{z^{3}}$ tip state inclined $37^{\circ}$ with respect to the $z$ axis. The calculation is based on the modified Bardeen approach (Ref. 9), in which the cantilever oscillation (amplitude $A=0.5 \mathrm{~nm}$ ) is accounted for. Average tunneling current $I_{a v}=1 \mathrm{nA}$, sample bias: $-100 \mathrm{mV}$. The color scales in (c) and (d) are identical. (e) Trace through the maximum of the image in the $x$ direction (red line). The paraboloid fitted to the trace has an apex radius of $R_{x}=0.12 \mathrm{~nm}$. (f) Trace through the maximum of the image in the $y$ direction (green line). The paraboloid fitted to the trace has an apex radius of $R_{y}=0.15 \mathrm{~nm}$. The black lines in (e) and (f) show the corresponding cross sections of the constant current surface at the main peak of the theoretical image. For clarity, the theoretical line scans are displaced in the $z$ direction. 
calculated image using the "modified Bardeen approach,"9 which is based on the quantum-mechanical perturbation theory. For the many-electron-atoms silicon, cobalt, iron, and samarium, "Slater-type-orbitals" 23 were calculated as approximations for the electronic $3 p$ and $4 f$ states. The only free input parameter for the calculations is the electronic density of states at the Fermi level ${ }^{9}$ of the tip, which was set to 0.4 electrons per atom and electron volt as a typical value for metals. ${ }^{24}$ The main result of these calculations is the excellent agreement between the experiment and theoretical results for $\operatorname{Sm~} 4 f_{z^{3}}$ wave functions inclined $37^{\circ}$ with respect to the axis normal to the silicon surface. Figure 6(e) and 6(f) show contour lines in $x$ and $y$ directions for both experimental and calculated images. The calculation accounts for an oscillation of the cantilever with an amplitude of $A$ $=0.5 \mathrm{~nm}$ in $z$ direction. In experiments as well as in calculations, the resulting image is insensitive to amplitude $A$, as long as $A$ is in the range of $0.3-1.5 \mathrm{~nm}$. The difference between the experimental and theoretical images (a slight compression in the $z$ direction of the experimental image compared to the theoretical image) is explained with atomic relaxations. Molecular-dynamics calculations reveal that the repulsive interaction occuring at the lower turning point of the oscillation compresses the atom image in the $z$ direction, leading to an increased experimental atom radius. The excellent agreement between Figs. 6(c) and 6(d) implies that in this experiment a pure $4 f_{z^{3}}$ state has carried the major part of the tunneling current, and contributions of other $4 f$ and possibly $6 s$ states are negligible. Whether the high selectivity for the $4 f_{z^{3}}$ state is caused by the magnetic field generated by the permanent magnet or by crystal field splitting in the field of the neighboring atoms of the front atom remains to be studied. In standard STM experiments, much higher tunneling voltages than those in our experiment are usually applied, probably resulting in a contribution of atomic states of more than one symmetry type [see Figs. 3(a) and 3(b)]. In this case, it is likely that the contributions of the various orbitals add to a roughly spherical symmetry of the image. As another important result of the calculations, it was found that the oscillation of the tip favors a monotonic control signal for the $z$ control of the STM feedback. Therefore, an oscillating tip may avoid a tip crash and enable lateral movement of the probe during the imaging process in cases where imaging might be impossible using a static probe. For static tips, atomic resolution imaging in the contact mode should be degraded because of instabilities of the tip atom. It was impossible to obtain atomically resolved images with a sample bias of only $-100 \mathrm{mV}$ when reducing the cantilever amplitude below $0.2 \mathrm{~nm}$. Amplitudes considerably larger than the decay length of the tunneling current result in a lower signal, because then electron tunneling can only occur for a small fraction of the oscillation cycle. The large spring

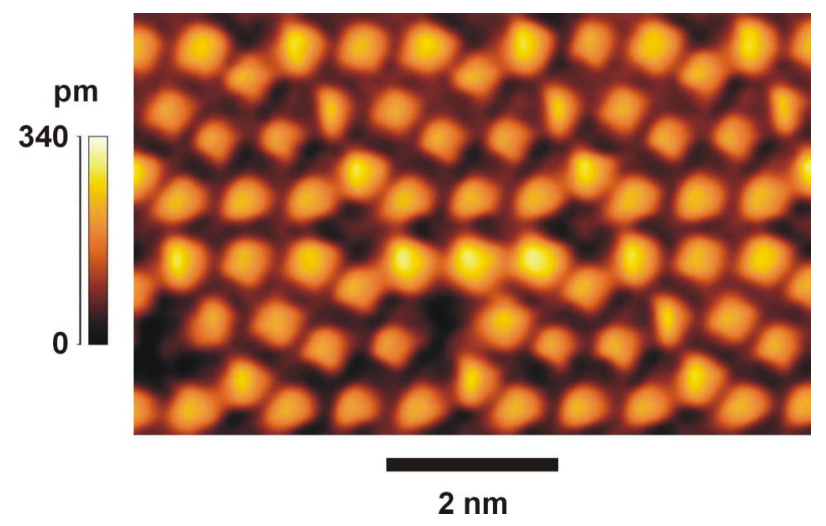

FIG. 7. (Color online) Experimental dynamic STM image of the $\mathrm{Si}(111)-(7 \times 7)$ surface using a samarium tip. Sample bias $-0.8 \mathrm{~V}$, tunneling current $I_{a v}=50 \mathrm{pA}$ with $A=0.8 \mathrm{~nm}$. Two single atomic defects are present.

constant of the cantilever used in our experiment assures stable operation at amplitudes in the range of interatomic distances.

In Fig. 7, dynamic STM data acquired with a samarium tip are shown. The special shapes of the atomic images, mainly with fourfold symmetry, are assumed to be the consequence of a convolution of the $s p^{3}$ silicon states with different samarium states protruding from a single samarium atom. The special atomic shapes are repeated in the adjacent unit cells according to the periodicity of the $\mathrm{Si}(111)$ $(7 \times 7)$ surface. Here, an interaction with the rest atoms cannot be detected. Single-atomic defects in adjacent Si unit cells prove that a single tip atom was responsible for the special atomic images.

In summary, we have found a subatomically varying transition probability for the tunneling process in STM, demonstrating the capability of the dynamic STM to image structures within atoms caused by atomic orbitals. The observability of the substructures is attributed to the dynamic STM mode with a cantilever operated at amplitudes $A$ in the range of $0.3 \mathrm{~nm} \lesssim A \lesssim 1.5 \mathrm{~nm}$ and a small tunneling bias voltage. Combining the dynamic STM with tip characterization tools such as field ion microscopy ${ }^{25}$ should further improve our understanding of the relation between atom shapes and tip states. Theoretical considerations link the experimental shape of the atoms to the atomic orbitals participating in the tunneling process. It is conceivable that chemical identification of the tip atom is possible with refined calculations of the tunneling current.

\section{ACKNOWLEDGMENTS}

We thank S. Hembacher, T. Kopp, C. Laschinger, and W. Scherer for valuable discussions. This work was supported by the Bundesministerium für Bildung und Forschung (Project No. EKM13N6918).

\footnotetext{
*Email address: Franz.Giessibl@physik.uni-augsburg.de

†URL: http://www.Physik.Uni-Augsburg.DE/exp6

${ }^{1}$ W. Heisenberg, Physics and Philosophy (Harper, New York, 1958), Chap. 4.
}

${ }^{2}$ P.A.M. Dirac, The Principles of Quantum Mechanics, 4th ed. (Clarendon, Oxford, 1967).

${ }^{3}$ G. Baym, Lectures on Quantum Mechanics (Benjamin, New York, 1969). 
${ }^{4}$ G. Schoknecht, Zeitschrift für Naturforschung, Part A, Astrophysik, Physik und Physikalische Chemie 12, 983 (1957).

${ }^{5}$ Y.W. Yang and P. Coppens, Solid State Commun. 15, 1555 (1974).

${ }^{6}$ J.M. Zuo, M. Kim, M. O'Keefe, and J.C.H. Spence, Nature (London) 401, 49 (1999).

${ }^{7}$ G. Binnig, H. Rohrer, Ch. Gerber, and E. Weibel, Phys. Rev. Lett. 49, 57 (1982).

${ }^{8}$ J. Tersoff and D.R. Hamann, Phys. Rev. Lett. 50, 1998 (1983).

${ }^{9}$ C.J. Chen, Introduction to Scanning Tunneling Microscopy (Oxford University Press, Oxford, New York, 1993).

${ }^{10}$ S.G. Wang and W.H.E. Schwarz, Angew. Chem., Int. Ed. Engl. 39, 3794 (2000).

${ }^{11}$ E. Scheer, N. Agrait, J.C. Cuevas, A. Levy Yeyati, B. Ludolph, A. Martin-Rodero, G. Rubio Bollinger, J.M. van Ruitenbeek, and C. Urbina, Nature (London) 394, 154 (1998).

${ }^{12}$ F.J. Giessibl, S. Hembacher, H. Bielefeldt, and J. Mannhart, Science 289, 422 (2000).

${ }^{13}$ F.J. Giessibl, H. Bielefeldt, S. Hembacher, and J. Mannhart, Ann. Phys. (Leipzig) 10, 887 (2001).

${ }^{14}$ Q. Zhong, D. Innis, K. Kjoller, and V.B. Elings, Surf. Sci. 290,
L688 (1993).

${ }^{15}$ M.R. Jarvis, R. Pérez, and M.C. Payne, Phys. Rev. Lett. 86, 1287 (2001).

${ }^{16}$ F.J. Giessibl, Appl. Phys. Lett. 73, 3956 (1998).

${ }^{17}$ U. Dürig, J.K. Gimzewski, and D.W. Pohl, Phys. Rev. Lett. 57, 2403 (1986).

${ }^{18}$ F.J. Giessibl and H. Bielefeldt, Phys. Rev. B 61, 9968 (2000).

${ }^{19}$ F.J. Giessibl, M. Herz, and J. Mannhart, Proc. Natl. Acad. Sci. U.S.A. 99, 12006 (2002).

${ }^{20}$ M.A. Lantz, H.J. Hug, P.J.A. van Schendel, R. Hoffmann, S. Martin, A. Baratoff, A. Abdurixit, H.-J. Güntherodt, and Ch. Gerber, Phys. Rev. Lett. 84, 2642 (2000).

${ }^{21}$ T. Eguchi and Y. Hasegawa, Phys. Rev. Lett. 89, 266105 (2002).

${ }^{22}$ W.A. Hofer, A.J. Fisher, R.A. Wolkow, and P. Grütter, Phys. Rev. Lett. 87, 236104 (2001).

${ }^{23}$ R. McWeeny, Coulson's Valence (Oxford University Press, Oxford, 1991), p. 42.

${ }^{24}$ N. Ashcroft and N. D. Mermin, Solid State Physics (Saunders College, Philadelphia, 1981).

${ }^{25}$ A. Schirmeisen, G. Cross, A. Stalder, P. Grütter, and U. Dürig, New J. Phys. 2, 29 (2000). 